\title{
Head injury in the elderly - an overview for the physician
}

\author{
Authors: William Beedham, ${ }^{\mathrm{A}}$ George Peck, ${ }^{\mathrm{B}}$ Simon E Richardson, ${ }^{\mathrm{C}}$ Kevin Tsang, ${ }^{\mathrm{D}}$ Michael Fertleman ${ }^{\mathrm{E}}$ and \\ David JH Shipway ${ }^{\mathrm{F}}$
}

Head injury is a common cause for hospital admission and additionally 250,000 UK inpatients fall during hospital admissions annually. Head injury most commonly occurs as a result of falls from standing height in older adults. Older adults are frequently frail and multi-morbid; many have indications for anticoagulation and antiplatelet agents. The haemorrhagic complications of head injury occur in up to $16 \%$ of anticoagulated patients sustaining a head injury. These patients suffer adverse outcomes from surgery as a result of medical complications. Although geriatric trauma models are evolving to meet the demand of an ageing trauma population, medical support to trauma services is commonly delivered by general physicians, many of whom lack experience and training in this field. Determining the role of surgery and interrupted anticoagulation requires careful personalised risk assessment. Appreciation of the opposing risks can be challenging; it requires an understanding of the evidence base in both surgery and medicine to rationalise decision making and inform communication. This article aims to provide an overview for the physician with clinical responsibility for patients who have sustained head injury.

KEYWORDS: Elderly, trauma, subdural, head injury, intracranial haemorrhage

\section{Introduction}

Head injury has historically been the domain of the surgeon. However, populations are ageing rapidly, and the number of persons aged over 85 is anticipated to double in forthcoming decades. ${ }^{1}$ In England and Wales there are 1.4 million hospital attendances due to head injury per annum, the majority of which occur in older persons due to low-energy falls from standing height. ${ }^{2}$

Authors: ${ }^{\text {A }}$ medical student, Birmingham University Medical School, Birmingham, UK; ${ }^{B}$ consultant physician and perioperative geriatrician, St Mary's Hospital, London, UK; ' Clinical lecturer in haematology, University College London, UK; ${ }^{\mathrm{D}}$ consultant neurosurgeon, St Mary's Hospital, London, UK; ${ }^{E}$ Consultant physician and perioperative geriatrician, St Mary's Hospital, London, UK; ' F consultant physician and perioperative geriatrician, Southmead Hospital, Bristol, UK
Between 2009 and 2015, the rate of patients being newly prescribed oral anticoagulation increased by $58 \%$, with over 500 per 100,000 people receiving oral anticoagulation prescriptions in 2015 . $^{3}$ Where head injury arises from falls in the context of anticoagulants or antiplatelet agents, haemorrhagic complications may be as high as $15.9 \%{ }^{4}$

Although neurosurgical teams are highly skilled in the management of haemorrhagic complications, age is associated with the accumulation of multi-morbidity. ${ }^{5}$ Multi-morbid patients challenge traditional surgical services because increasing super-specialisation has attenuated generalist skills. Adverse outcomes in older surgical patients are well-recognised, ${ }^{6}$ and these typically occur primarily as a consequence of medical complications of surgery. In the modern era, we need to recognise that traditional surgical services cannot deliver highquality medical care to complex patients without the support of medical teams.

Modern models of collaborative trauma care have evolved in orthopaedics; however these services are not yet ubiquitous. Impending changes to best practice tariffs for major trauma centres across England may soon incentivise diversion of geriatrician resource, but in many centres, medical support to trauma teams is provided by the duty medical registrar, who typically receives little (or no) dedicated training. Physicians are therefore frequently inadequately equipped to handle situations which are often complicated.

Furthermore, many haemorrhagic complications of head injury are sustained during complex medical admissions as a result of the 250,000 inpatient falls occurring annually in the UK. Trauma services do not have capacity to take over the care of all inpatients with complications of head injury, and typically only accept candidates for neurosurgery. Physicians therefore often have clinical responsibility for the care of patients with complications of head injury, yet lack knowledge and expertise to manage this cohort optimally.

There is therefore a need for better knowledge of head injury amongst physicians to provide more effective support to trauma services, and advocate for patients sustaining head injury during medical admissions.

\section{The effects of ageing on the brain}

A number of physiological changes occur with ageing that predispose older patients to haemorrhagic complications of head injury (Table 1). 
Table 1. Physiological changes of ageing and their clinical significance for head injury

\begin{tabular}{|c|c|}
\hline Physiological change & Clinical significance for head injury \\
\hline Cerebral atrophy & $\begin{array}{l}\text { Reduced brain parenchymal volume increases the space between the arachnoid } \\
\text { and dura mater. This leads to stretching of the bridging veins and vulnerability to } \\
\text { rupture. This is a common risk factor for subdural haemorrhage. The presence of } \\
\text { cerebral atrophy may also result in delayed presentation of altered consciousness } \\
\text { following traumatic brain injury, as a greater volume of blood is required to exert } \\
\text { pressure effects upon brain parenchyma. }\end{array}$ \\
\hline Hypertension & $\begin{array}{l}\text { Hypertension leads to increased wall tension within blood vessels. This is a risk factor } \\
\text { for aneurysm formation and blood vessel rupture, increasing the risk of subarachnoid } \\
\text { haemorrhage by up to } 180 \% .8\end{array}$ \\
\hline Reduced cerebral auto-regulation & $\begin{array}{l}\text { Impaired autoregulation of cerebral blood flow results in diminished blood supply } \\
\text { and hypoxic brain injury following head trauma. }\end{array}$ \\
\hline Cerebrovascular atherosclerosis & $\begin{array}{l}\text { Atherosclerosis contributes to the reduction in cerebrovascular autoregulation. } \\
\text { Atherosclerosis is associated with increased risk of spontaneous intracerebral } \\
\text { haemorrhage. }\end{array}$ \\
\hline Increased monoamine oxidase B concentration & $\begin{array}{l}\text { Monoamine oxidase B is responsible for de-amination of neurotransmitters. This } \\
\text { process produces reactive oxygen species (ROS) which cause cerebral damage. ROS } \\
\text { reduce the biological resilience of the brain, leaving the parenchyma vulnerable to } \\
\text { damage following injury. }^{9}\end{array}$ \\
\hline Ageing mitochondria & $\begin{array}{l}\text { Ageing mitochondria display delayed electron transport chain function and reduced } \\
\text { rate of adenosine triphosphate production. This reduces the cerebral resilience to } \\
\text { brain injury. }\end{array}$ \\
\hline Reduced superoxide dismutase concentrations & $\begin{array}{l}\text { Superoxide dismutase (SOD) is responsible for catalysing the partitioning of } \\
\text { superoxide radicals into hydrogen peroxide. Reduction in SOD leads to accumulation } \\
\text { of radicals and diminished resilience to brain injury. }{ }^{9}\end{array}$ \\
\hline Increased superoxide production & Superoxide leads to parenchymal damage and reduced injury resilience. ${ }^{9}$ \\
\hline
\end{tabular}

\section{Investigation}

\section{Indications for head imaging}

National Institute for Health and Care Excellence guidelines for head computed tomography (CT) are described in Table 2. ${ }^{2}$ However, evidence indicates that $30 \%$ of intracranial injuries do not present with reliable clinical findings. ${ }^{10}$ Atrophy of the ageing brain can allow older patients to tolerate substantial intracranial haemorrhage better than younger patients with equivalent injury. This can lead to underestimation of the extent or severity of injury in older persons and delayed presentation. Non-contrast CT head imaging may therefore be appropriate in all older patients presenting with significant head injury, especially if imaging outcome will influence medical decision making (eg prescribing of anticoagulants). It should be noted that Trauma Audit and Research Network (TARN) data indicate that older patients wait longer for head imaging than younger patients, despite being at higher risk of bleeding and or adverse outcomes. ${ }^{11}$

Most of the common haemorrhagic consequences of head injury can be adequately imaged by CT (Table 3), although magnetic resonance imaging (MRI) may have greater sensitivity for evaluating diffuse parenchymal damage, especially where small volume injury is not detected on plain CT. In patients on anticoagulation with initial normal CT, there is a small risk of subsequent scans demonstrating haemorrhage. Anti-coagulated patients may therefore benefit from a period of observation and repeat $\mathrm{CT}$ imaging prior to discharge. ${ }^{12}$

\section{Table 2. National Institute for Health and Care \\ Excellence guidelines for computed tomography head scan following head injury ${ }^{2}$}

Indications for CT head scan to be performed within 1 hour. Head injury and any one of:

$>$ GCS $<13$ on initial assessment

$>\mathrm{GCS}<152$ hours post-injury

$>$ suspected open/depressed skull fracture

signs of basal skull fracture

post-traumatic seizure

$>$ focal neurological signs

$>$ one plus episodes of vomiting since injury.

Indication for CT head scan to be performed within 8 hours. Head injury with any one of:

$>$ current anticoagulant treatment

$>$ loss of consciousness or amnesia since injury plus one of:

- age $>65$ years

- history of bleeding/clotting disorder

- dangerous mechanism of injury

- >30 min retrograde amnesia of events preceding injury.

$\mathrm{CT}$ = computed tomography; GCS = Glasgow coma score. 
Table 3. Consequences of head injury

Subdural haematoma

Cerebral contusions

Traumatic subarachnoid haemorrhage

Intracerebral haemorrhage

Extradural haemorrhage

Skull fractures

Diffuse axonal injury
Most common haemorrhage following elderly head injury. ${ }^{12}$

Characterised by bleeding into the space between the dura and arachnoid mater following acute arterial or parenchymal injury.

Most common aetiology of chronic subdural haematoma is tearing of bridging veins crossing the arachnoid and dura; risk exacerbated by cerebral atrophy

Bridging vein tears can also occur with intracranial hypotension (eg following CSF leak). ${ }^{13}$

Defined as clearly demarcated areas of small-volume focal micro-haemorrhage occurring as a direct result of damage to the small vessels of the brain parenchyma.

Can be located in proximity to the site of impact, but contrecoup distribution is common.

Results in focal neurological deficit. ${ }^{14}$

Commonly occurs due to direct injury to a blood vessel, or injury secondary to sudden increase in intravascular pressure. ${ }^{15}$

Characterised by bleeding into the CSF-filled subarachnoid space.

Can result in secondary obstructing hydrocephalus. ${ }^{15}$

Discrete collection of blood within the parenchyma of the brain.

Classified according to aetiology: trauma is less common than the primary spontaneous causes such as hypertension. ${ }^{16}$

Results in focal damage due to direct local compression or a more diffuse cerebral injury due to raised intracerebral pressure. ${ }^{16}$

Full haemorrhage formation can occur due to haemorrhagic progression of contusion. ${ }^{17}$

The least common post-traumatic intracranial haemorrhages. ${ }^{12}$

$75-90 \%$ of patients co-present with a skull fracture. ${ }^{18}$

The most common mechanism of injury is rupture of the middle meningeal artery as it passes through the foramen spinosum. ${ }^{19}$

Commonly accompanied by a characteristic period of lucidity following injury, which often precedes a comatose state.

Classified according to location, appearance and degree of depression. ${ }^{20}$

Can result in damage to brain parenchyma, blood vessels and cranial nerves as they exit the cranial vault.

Can also predispose to associated venous sinus thrombosis, especially in occipital fractures crossing the site of the transverse sinus. CT venography may be indicated in the context of persistent headaches or feature of raised intracranial pressure.

CSF leakage can result in intracranial hypotension and secondary subdural haemorrhage.

A syndrome representing microscopic damage to the axons of the neural tract, brainstem and corpus callosum.

Clinically defined as a coma lasting longer than 6 hours following a traumatic brain injury without other ischemic lesions or swelling identified as the underlying cause. ${ }^{21}$

CSF = cerebrospinal fluid; $\mathrm{CT}$ = computed tomography

\section{Management}

\section{Indications for neurosurgery}

The role of neurosurgery is to relieve elevated intracranial pressure (ICP), improve the cerebral perfusion pressure and correct depressed skull fractures. NICE guidelines state that the following signs indicate neurosurgical consultation:

$>$ unexplained confusion of $>4$ hours

persistent Glasgow coma score (GCS) $<8$

$>$ deteriorating GCS after admission

$>$ development of focal neurological signs
$>$ seizure without full recovery

$>$ penetrating head injury

$>$ cerebrospinal fluid (CSF) leak. ${ }^{2}$

North American consensus guidelines indicate that in patients with potential to benefit, surgical evacuation of intracranial haemorrhage should be considered (regardless of GCS) where radiological parameters show:

> either subdural haematoma with $10 \mathrm{~mm}+$ clot thickness or $5 \mathrm{~mm}+$ midline shift $^{22}$

> or epidural haematoma with volume $>30 \mathrm{~cm}^{3}$. 
Surgery may also be indicated where these criteria are not met, and there remains clinical evidence of brain herniation or raised ICP (eg anisocoria), especially in the context of documented clinical deterioration. Surgical outcomes may be dependent on time between onset of neurological signs and surgical decompression. Where surgery is indicated, it should take place within 4 hours.

In patients not meeting surgical criteria but with GCS $<9$, ICP monitoring in a critical care setting is advised. Surgery should be considered if ICPs are sustained above $20 \mathrm{mmHg}$. There exists considerable debate and controversy as to the indication and outcome of decompressive neurosurgery in these patients who do not fit the haematoma criteria above. Various studies suggest that these patients often have improved survival but potentially worse neurological outcomes, and a role for decompressive craniectomy in patients with advanced frailty is generally less accepted.

\section{Non-operative management}

Non-operative management of raised ICP is appropriate when there is a small haematoma, or the intracerebral pressure is below $20 \mathrm{mmHg} .{ }^{22}$ Non-surgical interventions include head elevation, sedation, analgesia, mechanical hyperventilation to maintain normal $\mathrm{pCO}_{2}$, euthermia, antiepileptics, mannitol (or hypertonic saline) and ICP monitoring. ${ }^{24}$ In patients who are potential surgical candidates, the risk of haematoma expansion indicates serial CT 8-12 hours after injury. ${ }^{25}$ For ICP monitoring, ventricular catheters are considered the gold standard in the USA. These devices permit therapeutic CSF drainage if required, although they do carry a greater infection risk. In the UK intraparenchymal monitoring devices are more commonly deployed. ${ }^{26}$

Outcomes from intracranial haemorrhage have been shown to be superior in patients transferred to a specialist neurology or neurosurgical unit, even if surgical intervention is not required immediately. ${ }^{27}$ In symptomatic patients who would be good surgical candidates should they deteriorate, transfer to a neurosurgical centre may therefore be appropriate for monitoring and medical management (eg for severe hypertension).

\section{Neurosurgery in the older patient}

Despite the availability of consensus guidelines, older patients are less likely to undergo neurosurgical intervention than younger patients with equivalent injury. This may be because older patients treated surgically have inferior outcomes. ${ }^{28}$ In one study, survival following surgical intervention for traumatic head injury was $59.3 \%$ for ages $65-74$ and only $32.4 \%$ for those aged over $75 .^{29}$ However, compared to age matched peers treated conservatively, older persons undergoing surgery following head trauma had significantly reduced mortality and better functional outcomes. ${ }^{28}$ Debate persists about the benefits and role of decompressive neurosurgery in the older patient with haemorrhagic complications of head injury. This is complicated by difficulties interpreting the literature when it is known that older patients tend to tolerate more substantial intracranial injury before reflecting the severity of their injury in their GCS. Adverse outcomes in older patients with matched GCS to younger patients may therefore represent more severe injury and unfair comparison.

Balancing risk and benefit in older patients with complications of head injury is therefore complex. Careful consideration of likely post-operative outcome is required on an individual basis. Age and frailty are not synonymous, and wide functional heterogeneity can be found in older persons. Emerging evidence indicates that frailty assessment provides a more accurate prediction of surgical risk than age, and frailty seems to intuitively influence the decision to operate in patients undergoing oncological neurosurgery. ${ }^{30}$ There is some evidence that frailty is associated with poor outcomes in geriatric trauma, but little published data specific to elderly traumatic brain injury. Sarcopaenia defined by CT criteria is emerging as a potential surrogate for frailty assessment that may prove useful in predicting outcome in the future. ${ }^{31}$

\section{Role of the physician}

Importantly, the UK hub and spoke trauma model means that many decisions on whether to operate are currently made without the direct assessment of an experienced neurosurgeon. The decision on whether or not to offer surgery therefore depends on information-gathering and communication in relation to baseline state. Combined with injury severity, this determines potential for recovery and allows neurosurgical prognostication. However, subsequent communication with patient and family is often the responsibility of the referring medical team, especially where surgery is not indicated. This emphasises the need for physicians to understand the basis and rationale for decision-making.

In addition to review of mechanism of injury and optimisation of co-morbidity, the responsibility of the physicians is also to treat complications and promote rehabilitation. Management of key medical issues is discussed in the following paragraphs.

\section{Management of anticoagulation}

Many older persons regularly take anticoagulants and antiplatelet agents which contribute to haemorrhagic complications of head injury. Challenging decisions are often required pertaining to the reversal of anticoagulant and antiplatelet effect. Timed reintroduction of these drugs following haemorrhagic complications of head injury is also complex.

In these cases, judicious assessment of risk is required. The risk of thrombosis in each individual must be balanced against the risks of recurrent intracranial bleeding. These risks are often difficult to estimate for general physicians without experience in the management of traumatic head injury. This can lead to poor decision-making if there are delays in obtaining specialist advice.

Mortality from haemorrhagic complications of head injury in the presence of continued anticoagulation is strikingly elevated, and may reach $80.6 \%{ }^{32}$ The risks associated with cessation of anticoagulation are generally extrapolated from annualised population risks (Table 4) and therefore may be higher in the acute setting. Notwithstanding this observation, the risks of thrombosis are usually substantially lower than that of the acute bleed, even for patients considered at high risk of thrombosis. Suspension of anticoagulation is therefore almost always required; this should be discussed with a haematologist with specialist coagulation experience. A risk-benefit analysis and management plan should be clearly documented and regularly reviewed.

\section{Reversal of anticoagulants}

NICE recommends that warfarinised patients presenting with suspected or confirmed intracranial bleeding should undergo immediate reversal with prothrombin complex concentrate (PCC). ${ }^{40}$ This is supported by the British Committee for Standards 
Table 4. Risk of thrombosis in common indications for anticoagulation

Atrial fibrillation

Venous thromboembolism

Mechanical heart valves

Lupus anticoagulant

Myeloproliferative disorders
A 2013 study found that cessation of anticoagulation in atrial fibrillation patients was associated with a $5.17 \%$ per year risk of thrombotic event. Adjusted analysis revealed that cessation resulted 3.43-fold risk of death ${ }^{33}$ and overall thrombosis risk of $0.5 / 1000$ cases off treatment for 5 days. The $\mathrm{CHA}_{2} \mathrm{DS}_{2}-\mathrm{VASC}$ score is currently recommended to calculate individualised atrial fibrillation-associated stroke risk ${ }^{34}$ and should be documented - those with a score of over 3 are considered to be at higher risk of thrombosis (1.6/1000 patients for 5 days off anticoagulation).

Patients at highest risk are those within 3 months of a VTE (in particular within 6 weeks) or who have previously developed VTE on anticoagulation (eg treated with an international normalised ratio target 3-4). Management of anticoagulation should be discussed with haematology.

Patients who develop provoked VTE are generally considered to be at lower risk of recurrence after cessation of anticoagulation and would not normally require long-term anticoagulation. ${ }^{35}$ Conversely, a large meta-analysis found significantly higher recurrence rates of PE and DVT in patients whose VTE were unprovoked; ${ }^{36}$ with $22.6 \%$ recurrence at 5 years after cessation of anticoagulation.

Overall, patients at increased risk include those with unprovoked or recurrent VTE, presentation with PE and those with on-going risk factors (eg active cancer). The management of anticoagulation in such patients should be discussed with haematology and consideration given to non-pharmacological VTE prophylaxis (eg good hydration, early mobilisation, compression stockings) and the use of an inferior vena cava filter in selected cases with active lower limb DVT at risk of embolisation.

The short-term risk of thrombosis is generally overstated and varies with the type of valve (bileaflet less than tilting disc or caged ball); ${ }^{37}$ valve position ( $\mathrm{AV}<\mathrm{MV}$ ); additional patient risk factors (previous TIA/ CVA, atrial fibrillation, impaired left ventricular ejection fraction).

A large meta-analysis of RCTs and observational studies concluded that the total risk of thromboembolism without anti-coagulation of these patients is 8.6 events per 100 patient years. ${ }^{37}$

Patients with a bileaflet aortic valve and no additional risk factors are at relatively low risk (0.5/1000 risk for 5 days off anticoagulation), whereas in those with $\mathrm{MV}$ replacements and risk factors the risk is higher (1.6/1000 for 5 days off anticoagulation); specialist advice should be sought.

The use of unfractionated heparin is generally not recommended due to the thrombotic and bleeding risks associated with limited time in range. If bridging is required LMWH is generally advised, but importantly, warfarin is by far the most effective anticoagulant agent in mechanical heart valve setting.

A 2015 study found that there was a risk of 0.532 events per 100 patient years who suffer with systemic lupus erythematosus when anti-coagulated. ${ }^{38}$

Patients with antiphospholipid syndrome with a known thrombotic history can exhibit aggressive thrombotic tendencies, often complicated by co-existent thrombocytopenia. The management of these rare, but complex patients requires individualised assessment by a haematologist with experience in coagulation disorders.

Polycythaemia vera and essential thrombocytosis carry a significant thromboembolic risk including arterial thrombosis and a tendency to splanchnic and cerebral vein thromboses. A 2014 study found that patients with polycythaemia vera had a thromboembolic risk of $2.5-5 \%$ per patient year, with essential thrombocythaemia patients having a similar 1.9-3\% risk of event per patient year. ${ }^{39}$

Thrombotic risk is highly variable between patients and will depend on the age, thrombotic history and overall blood count picture at the time (including white cell counts). Most MPD patients are given primary prophylaxis with aspirin, which can be difficult to fully reverse, and such patients should be discussed with haematology.

$\mathrm{AV}=$ aortic valve; $\mathrm{CVA}=$ cerebrovascular accident; $\mathrm{DVT}=$ deep vein thrombosis; $\mathrm{LMWH}=$ low molecular weight heparin; $\mathrm{MPD}=\mathrm{myeloproliferative} \mathrm{disorders;} \mathrm{MV}=$ mitral valve; $\mathrm{PE}=$ pulmonary embolism; RCTs = randomised controlled trials; TIA = transient ischemic attack; VTE = venous thromboembolism

in Haematology, which states that following head-injury admission anticoagulated patients are likely to require a CT scan, need their international normalised ratio (INR) measured, and if head injury is strongly suspected reversal of the INR with PCC. ${ }^{41}$ The use of recombinant factor VIIa or fresh frozen plasma is not acceptable management. ${ }^{42}$ Further measures should include $5-10 \mathrm{mg}$ of intravenous vitamin $\mathrm{K}$, immediate cessation of all anticoagulant therapy and serial measurement of INR at
30 minutes, $4-6$ hours and 24 hours in case further doses of PCC are required.

The reversal of direct oral anticoagulants (DOACs) is more complex. The factor II inhibitor dabigatran can be effectively reversed with idarucizumab. Andexenant, a specific reversal agent for factor $X$ inhibitors, is in development. Meanwhile, expert consensus currently advises that suspected intracranial bleeding in patients taking the commonly prescribed factor $X$ inhibiting 
DOACs, be reversed with PCC, although this is unlikely to be fully effective. ${ }^{43}$ Tranexamic acid can be used adjunctively and carries limited risk of thrombosis.

\section{Reversal of antiplatelet effect}

Some evidence indicates that clopidogrel may predispose to traumatic intracranial haemorrhage to a greater extent than warfarin. Limited evidence is available to determine the risks of modern potent antiplatelet agents. Strategies for reversal of antiplatelet effect include regular tranexamic acid, platelet infusion (typically 2 pools) and in extreme cases recombinant-activated factor VIIa. Of note, whilst two pools of platelets would be expected to largely reverse aspirin effect, the prolonged pharmacodynamics of clopidogrel (5-7 day washout) limits the efficacy of platelet transfusion and multiple pools are often required. However, none of these interventions improves outcome following intracerebral haemorrhage. ${ }^{44}$ Suspension of antiplatelet agents following acute intracranial haemorrhage is however advised.

\section{Restarting anticoagulants and antiplatelets}

Although haematoma expansion and re-bleeding are significant risks following intracerebral haemorrhage, there is also an increased risk of thromboembolism precipitated by immobility, the inflammatory response to trauma and pro-coagulant effects of reversal agents. Non-pharmacological VTE prophylaxis should be instigated and understanding this evolving risk profile permits timely reinstatement of anti-coagulation therapy where thrombosis risk is deemed high. ${ }^{45}$

In patients with high thrombosis risk, current evidence indicates that anticoagulant therapy can be reinstated after 10 days with modest risk of re-bleeding. However, the risks of reinstating anticoagulation will vary depending on the extent and site of intracranial bleeding. There is no current consensus on what constitutes an ideal interval, and prospective studies to answer this question are currently underway. Many surgeons prefer to delay reinstating anticoagulation until $\mathrm{CT}$ brain indicates complete resolution of haemorrhage. Inevitably, this requires judicious appreciation of the risks of continued suspension of anticoagulation. However, it should also be noted that the risks of interrupted anticoagulant therapy are modest even in the context of modern metallic heart valves, where valve thrombosis rates of $4 \%$ per annum are seen in patients not taking anticoagulants. ${ }^{37}$ Outcomes for patients with metallic heart valves whose anticoagulation was suspended report a variable thrombosis risk $0-4 \%$ at 30 days. $^{46}$

Guidelines recommend 7-10 days of suspended anti-coagulation following haemorrhagic complications of head injury. ${ }^{47}$ Evidence pertaining to the reintroduction of DOACs following haemorrhagic head injury is limited, but re-bleeding following intracerebral haemorrhage is rarer on DOACs than warfarin and likely to be safer. ${ }^{48}$ Expert consensus therefore also recommends restarting DOACs following 7-10 days of suspension where thrombosis risk is high. The evidence surrounding reinstatement of antiplatelet agents is limited. However, anti-platelet agents are safer than anti-coagulants after intracerebral haemorrhage. Expert consensus indicates a 7-10 day cessation period may therefore be appropriate. $^{49}$

For patients at extremely high risk of thrombosis in whom it is thought early re-anticoagulation is indicated $<7$ days, or where re-bleeding risk is considered higher, graduated re-introduction of escalating doses of twice per day (bd) low molecular weight heparin (LMWH) is standard practice. This is generally considered safer and more effective than unfractionated heparin. Of note, in the case of re-bleeding, up to $60 \%$ of LMWH effect can be antagonised by protamine. Embolisation from recent lower limb venous thrombosis may be mitigated by IVC filter insertion.

\section{Management of thrombocytopaenia}

Thrombocytopaenia carries a $>12$-fold risk of haematoma expansion. ${ }^{49}$ Patients presenting with a platelet count $<135 \times 10^{9} / \mathrm{L}$ are 31.5 times more likely to require neurosurgical intervention. Thrombocytopenic patients with intracranial haemorrhage should receive platelet transfusion to maintain platelets $>100 \times 10^{9} / \mathrm{L} .^{49}$

\section{Seizures}

\section{Prophylaxis}

Early seizures occur in $30 \%$ of patients following traumatic brain injury. Most seizures occur within the first 24 hours of injury. Seizures are believed to increase the risks of secondary brain injury through functional hypoxia and raised ICP. Prophylaxis with anticonvulsants has been shown to reduce the frequency of early seizures, although anticonvulsants have no effect on the development of long-term epilepsy after brain injury. A recent survey of neurosurgical centres in the UK suggests that $50 \%$ of neurosurgical centres routinely use anticonvulsants for seizure prophylaxis after brain injury. ${ }^{50}$ Current evidence indicates that levetiracetam has a better safety profile and equal efficacy to phenytoin. Levetiracetam may also be associated with better functional outcomes. Prophylaxis with levetiracetam 500 $1000 \mathrm{mg}$ bd for 7 days is therefore recommended. ${ }^{51}$ In patients who have not suffered a seizure during this period, there is no advantage to long-term anticonvulsant therapy. In elderly patients, lower dosages should be considered and it is important that duration of therapy is confirmed prior to discharge.

\section{Treatment}

In patients sustaining seizures following traumatic brain injury, the risk of long-term seizures depends upon injury severity. Approximately $8-16 \%$ will suffer from post-traumatic epilepsy at 2 years. Additionally, patients with persistently reduced consciousness considered disproportionate to the extent of neurological injury, 15-20\% will display signs of non-convulsive seizures on continuous electroencephalography. ${ }^{52}$ Anticonvulsants are typically continued throughout the admission for patients who have sustained a seizure, and weaned gradually after discharge under neurological supervision.

\section{Hyponatraemia}

Hyponatraemia is a common complication of intracranial injury. Careful clinical evaluation is required to differentiate between the syndromes of inappropriate antidiuretic hormone secretion (SIADH), cerebral salt wasting (CSW) and other causes such as hypoadrenalism or drugs. SIADH is the most common electrolyte disturbance following head injury, but may be mistaken for CSW. The pathophysiology of CSW is poorly understood although it is known to result in excess renal excretion of sodium and dehydration. Evaluation of hyponatraemia is complicated by the fact that SIADH and CSW often display similar laboratory 
parameters. However, treatment is diametrically opposed and incorrect treatment risks deterioration in hyponatraemia. Differentiation therefore mandates careful evaluation of fluid status. Patients suffering from CSW are intravascularly deplete and require intravenous volume replacement; patients with SIADH are euvolaemic and require fluid restriction. Resolution of CSW typically occurs within $2-4$ weeks of head injury. ${ }^{53}$

\section{Prognosis}

Elderly patients suffer high mortality and reduced functional outcome following head injury when compared to younger cohorts; a recent UK cohort study reported $22.9 \%$ inpatient mortality, $10.8 \%$ long-term moderate disability and $5.3 \%$ severe disability requiring permanent help with activities of daily living. ${ }^{54}$ Outcomes are considerably worse for severe traumatic brain injury with inpatient mortality as high as $70-80 \%$.

\section{Conclusions}

Physicians are increasingly called upon to provide medical support for trauma services, whose population is increasingly old, frail and comorbid. Frail older patients often sustain head injury when they fall, and are predisposed to haemorrhagic complications because of anticoagulant use and the effects of ageing. Determining the role of surgery and interrupted anticoagulation requires careful, personalised risk assessment. Appreciation of the opposing risks can be challenging; it requires an understanding of the evidence base in both surgery and medicine to rationalise decision making and inform bilateral communication. .

\section{References}

1 Office for National Statistics. National population projections: 2014-based statistical bulletin. Office for National Statistics, 2015.

2 National Institute for Health and Care Excellence. Head injury: assessment and early management. Clinical guideline [CG176]. NICE, 2014.

3 Loo S, Dell'Aniello S, Huiart L, Renoux C. Trends in the prescription of novel oral anticoagulants in UK primary care. $\mathrm{Br}$ ] Clin Pharmacol 2017:83:2096-106.

4 Alrajhi K, Perry J, Forster A. Intracranial bleeds after minor and minimal head injury in patients on warfarin. J Emerg Med 2015:48:137-42

5 Department of Health. Long term conditions compendium of information: third edition. DH, 2012

6 Qureshi A, Mendelow A, Hanley D. Intracerebral haemorrhage. Lancet 2009;373:1632-44.

7 Adhiyaman V. Chronic subdural haematoma in the elderly. Postgrad Med J 2002;78:71-5.

8 Teunissen L, Rinkel G, Algra A, van Gijn J. Risk factors for subarachnoid hemorrhage: a systematic review. Stroke 1996;27:544-9.

9 Tobe EH. Geriatric traumatic brain injury: relationship to dementia and neurodegenerative disease. J Gerontol Geriatr Res 2016;5:292.

10 Mack L, Chan S, Silva J, Hogan T. The use of head computed tomography in elderly patients sustaining minor head trauma. J Emerg Med 2003:24:157-62.

11 The Trauma Audit and Research Network (TARN). Major Trauma in Older People. Salford: The University of Manchester; TARN, 2017.

12 Doherty DL. Post-traumatic cerebral atrophy as a risk factor for delayed acute subdural haemorrhage. Arch Phys Med Rehabil 1988;69:542.
13 Zare M, Ahmadi K, Zadegan S, Farsi D, Rahimi-Movaghar V. Effects of brain contusion on mild traumatic brain-injured patients. Int J Neurosci 2012;123:65-9.

14 Agrawal M, Modi N, Sinha V. Post-traumatic subarachnoid hemorrhage: A review. Neurol India 2016;64:8.

15 Jauch E, Elias B. Intracerebral haemorrhage pathophysiology and management. Air Med J 1999;18:62-7.

16 Kurland D, Hong C, Aarabi B, Gerzanich V, Simard J. Hemorrhagic progression of a contusion after traumatic brain injury: a review. J Neurotrauma 2012;29:19-31.

17 Talbott JF, Gean A, Yuh EL, Stiver SI. Calvarial fracture patterns on $\mathrm{CT}$ imaging predict risk of a delayed epidural hematoma following decompressive craniectomy for traumatic brain injury. AJNR Am J Neuroradiol 2014:35:1930.

18 Gean AD, Fischbein NJ, Purcell DD et al. Benign anterior temporal epidural hematoma: indolent lesion with a characteristic CT imaging appearance after blunt head trauma. Radiology 2010;257:212-8.

19 Demetriades D, Kobayashi L. Skull fractures. BMJ, 2017. http://bestpractice.bmj.com/topics/en-gb/398 [Accessed 03 May 2018].

20 Vieira R, Paiva W, de Oliveira D et al. Diffuse axonal injury: epidemiology, outcome and associated risk factors. Front Neurol 2016:7:178.

21 Bullock M, Chesnut R, Ghajar ] et al. Surgical management of acute subdural hematomas. Neurosurgery 2006;58(Suppl 3):S16-24.

22 Bullock M, Chesnut R, Ghajar ] et al. Surgical management of acute epidural hematomas. Neurosurgery 2006;58(Suppl 3):S7-15.

23 Ragland J, Lee K. Critical care management and monitoring of intracranial pressure. J Neurocrit Care 2016;9:105-12.

24 Sumritpradit P, Setthalikhit T, Chumnanvej S. Assessment and predicting factors of repeated brain computed tomography in traumatic brain injury patients for risk-stratified care management: a 5-year retrospective study. Neurol Res Int 2016;2016:1-7.

25 Tavakoli S, Peitz G, Ares W, Hafeez S, Grandhi R. Complications of invasive intracranial pressure monitoring devices in neurocritical care. Neurosurg Focus 2017;43:E6.

26 Diringer M, Edwards D. Admission to a neurologic/neurosurgical intensive care unit is associated with reduced mortality rate after intracerebral hemorrhage. Crit Care Med 2001;29:635-40.

27 Shimoda K, Maeda T, Tado M et al. Outcome and surgical management for geriatric traumatic brain injury: analysis of 888 cases registered in the Japan neurotrauma data bank. World Neurosurg 2014:82:1300-6.

28 Leung G, Lai-Fung L, Wai-Man L, Wong H, Yuen W. Outcome after operative intervention for traumatic brain injuries in the elderly. Asian J Neurosurg 2017;12:37.

29 Youngerman BE, Neugut AI, Yang ] et al. The modified frailty index and 30-day adverse events in oncologic neurosurgery. J Neurooncol 2018;136:197-206

30 Hu P, Uhlich R, White J, Kerby J, Bosarge P. Sarcopenia measured using masseter area predicts early mortality following severe traumatic brain injury. J Neurotrauma 2018;35:2400-6.

31 Cohen D, Rinker C, Wilberger J. Traumatic brain injury in anticoagulated patients. J Trauma 2006;60:553-7.

32 Bogseth $\mathrm{M}$. The significance of platelet count in traumatic brain injury patients on antiplatelet therapy. J Emerg Med 2015;48:262-3.

33 National Institute for Health and Care Excellence. Atrial fibrillation: management. Clinical guideline [CG180]. NICE, 2014.

$34 \mathrm{Kim}$ J, Khavanin N, Rambachan A et al. Surgical duration and risk of venous thromboembolism. JAMA Surgery 2015;150:110.

35 Baglin T, Douketis J, Tosetto A et al. Does the clinical presentation and extent of venous thrombosis predict likelihood and type of recurrence? A patient-level meta-analysis. J Thromb Haemost 2010;8:2436-42. 
36 Cannegieter S, Rosendaal F, Briet E. Thromboembolic and bleeding complications in patients with mechanical heart valve prostheses. Circulation 1994;89:635-41.

37 Aviña-Zubieta J, Lacaille D, Sayre E et al. Risk of pulmonary embolism and deep vein thrombosis in systemic lupus erythematosus: a population-based cohort study. Arthritis Res Ther 2012;14(Suppl 3):A53.

38 Kreher S, Ochsenreither S, Trappe R et al. Prophylaxis and management of venous thromboembolism in patients with myeloproliferative neoplasms: consensus statement of the Haemostasis Working Party of the German Society of Hematology and Oncology (DGHO), the Austrian Society of Hematology and Oncology (ÖGHO) and Society of Thrombosis and Haemostasis Research (GTH eV). Ann Hematol 2014;93:1953-63.

39 National Institute for Health and Care Excellence. Blood transfusion NICE guideline [NG24]. NICE, 2015.

40 Keeling D, Baglin T, Tait $C$ et al. Guidelines on oral anticoagulation with warfarin - fourth edition. Br J Haematol 2011;154:311-24.

41 Frontera J, Lewin J, Rabinstein A et al. Guideline for reversal of antithrombotics in intracranial hemorrhage. Crit Care Med 2016:44:2251-7.

42 Steiner T, Weitz J, Veltkamp R. Anticoagulant-associated intracranial hemorrhage in the era of reversal agents. Stroke 2017:48:1432-7.

43 Jaben E, Mulay S, Stubbs J. Reversing the effects of antiplatelet agents in the setting of intracranial hemorrhage. J Intensive Care Med 2014;30:3-7.

44 Murthy S, Gupta A, Merkler A et al. Restarting anticoagulant therapy after intracranial hemorrhage. Stroke 2017:48:15941600.

45 Panduranga P. Management dilemmas in patients with mechanical heart valves and warfarin-induced major bleeding. World ] Cardiol 2012;4:54.
46 Broderick J, Connolly S, Feldmann E et al. Guidelines for the management of spontaneous intracerebral hemorrhage in adults: 2007 update: a guideline from the American Heart Association/ American Stroke Association Stroke Council, High Blood Pressure Research Council, and the Quality of Care and Outcomes in Research Interdisciplinary Working Group: The American Academy of Neurology affirms the value of this guideline as an educational tool for neurologists. Stroke 2007;38:2001-23.

47 Milling T, Spyropoulos A. re-initiation of dabigatran and direct factor Xa antagonists after a major bleed. Am J Med 2016;129:S54-63.

48 Goldstein J, Greenberg S. Should anticoagulation be resumed after intracerebral hemorrhage? Cleve Clin J Med 2010;77:791-9.

49 Bogseth $\mathrm{M}$. The significance of platelet count in traumatic brain injury patients on antiplatelet therapy. J Emerg Med 2015;48:262-3.

50 Mee H, Kolias AG, Chari A et al. Pharmacological management of post-traumatic seizures in adults: current practice patterns in the UK and the Republic of Ireland. Acta Neurochir (Wien) 2018.

$51 \mathrm{Xu}$ JC, Shen J, Shao WZ et al. The safety and efficacy of levetiracetam versus phenytoin for seizure prophylaxis after traumatic brain injury: A systematic review and meta-analysis. Brain Inj 2016;30:1054-61.

52 Zimmermann LL, Diaz-Arrastia R, Vespa PM. Seizures and the role of anticonvulsants after traumatic brain injury. Neurosurg Clin N Am 2016;27:499.

53 Verbalis JG. Hyponatremia with intracranial disease: not often cerebral salt wasting. J Clin Endocrinol Metab 2014;99:59-62.

54 Hawley C, Sakr M, Scapinello S, Salvo J, Wrenn P. Traumatic brain injuries in older adults -6 years of data for one UK trauma centre: retrospective analysis of prospectively collected data. J Emerg Med 2017:34:509-16.

Address for correspondence: Dr David Shipway, Southmead Hospital, North Bristol NHS Trust, Bristol BS10 5NB, UK. Email: shipway@doctors.org.uk 